\title{
The Impact of Age on the Likely Impact of Coronary Calcium Testing in the 2018 Cholesterol Guidelines
}

\author{
Jeremy B. Sussman, MD, MS $S^{1,2,3}$ (i) and Venkatesh L. Murthy, MD, $P H D^{3,4}$ \\ 'Division of General Medicine, University of Michigan, Ann Arbor, MI, USA; ${ }^{2}$ Center for Clinical Management Research, VA Ann Arbor Healthcare \\ System, University of Michigan, Ann Arbor, MI, USA; ${ }^{3}$ Institute for Healthcare Policy and Innovation, University of Michigan, Ann Arbor, MI, USA; \\ ${ }^{4}$ Division of Cardiovascular Medicine and Frankel Cardiovascular Center, University of Michigan, Ann Arbor, MI, USA.
}

J Gen Intern Med 35(1):386-8

DOI: $10.1007 / \mathrm{s} 11606-019-05369-\mathrm{w}$

(c) Society of General Internal Medicine 2019

\section{BACKGROUND}

The 2018 American College of Cardiology/American Heart Association cholesterol guidelines recommend substantially increasing use of coronary artery calcium testing. ${ }^{1}$ In particular, they say it is appropriate for interested individuals aged 45 to 75 with a 10-year risk of atherosclerotic cardiovascular disease (ASCVD) between 7.5 and 20\%, who do not smoke, have diabetes, or already use a statin. If a patient has a CAC of 0 , the guidelines advise that statin use is not necessary.

However, calcification and ASCVD risk both increase with age. $^{2}$ The implications of this on the appropriateness of imaging are not known. We sought to clarify how many people this recommendation might affect and how aging might alter the potential impact of this recommendation on treatment choices.

\section{METHODS}

We used data from the Multi-Ethnic Study of Atherosclerosis (MESA), a large, community-based prospective cohort study of heart disease. ${ }^{3}$ We identified the subgroup of MESA participants who CAC testing would be considered appropriate in the 2018 cholesterol guidelines. We developed cubic splines fitted on age to address nonlinearities. We performed logistic regression then postestimation prediction using the cubic splines to estimate the likelihood a patient coronary calcium screening will be eligible for testing at each age. We used fractional polynomial regression of the relationship between age and the numbers of risk factors to be eligible for testing to learn how many risk factors would have to be positive for a patient to be appropriate at each age. We then examined the likelihood a test will have a CAC of 0 at each age.

This work has not been presented publically.

Electronic supplementary material The online version of this article (https://doi.org/10.1007/s11606-019-05369-w) contains supplementary material, which is available to authorized users.

Received June 21, 2019

Revised July 26, 2019

Accepted September 12, 2019

Published online November 8, 2019
This study received exemption from the Michigan Medicine IRB and we were given data access from BioLincc. All code is available at https://github.com/jeremysussman/ cacAge. This study received exemption from the Michigan Medicine IRB.

\section{RESULTS}

Of 6745 participants in the MESA study with a CAC scan, 808 subjects were outside of the guideline recommended age range of 45-75. A total of 3879 had a 10-year ASCVD risk outside the range of 7.5 to $20 \%$ recommended for testing. Eight hundred seven-three of the remaining patients either had diabetes, were current smokers, were on any lipid-lowering medicine, or had an LDL > 190. Consequently, only $17 \%(N=$ 1185 ) are eligible for CAC testing according to the guidelines (Fig. 1). CAC testing is considered appropriate for only $15 \%$ of 45 -year-olds. The rate of appropriate testing is highest at age 67 , at $37 \%$. CAC testing will be guideline-appropriate for under $1 \%$ of women under age 55 in MESA.] $\rightarrow$

The number of appropriate CAC scans with no calcium, which are the only ones that alter an individual's treatment recommendation, will decline dramatically across the age range (Fig. 2). At age 45 , we estimate $80 \%$ will have a CAC of 0 . By age 75 , it is under $40 \%$.] $\rightarrow$

\section{CONCLUSION}

Coronary calcium screening could help risk refinement for millions of Americans. Here, we found that the likelihood a patient will be guideline-eligible, the risk factor profile of those who are, and the likelihood the test will lead to a change in treatment recommendation change dramatically with age. For no age will eligibility exceed $40 \%$ of the population and under $20 \%$ are eligible until age 60 . Limitations of this study include the non-representative nature of MESA data. By the current guidelines, CAC screening will rarely be appropriate for younger people and only in those with multiple risk factors, but in younger patients, the results will be much more likely to be zero. These findings will likely impact the public health impact, effectiveness, and cost-effectiveness of CAC test. 


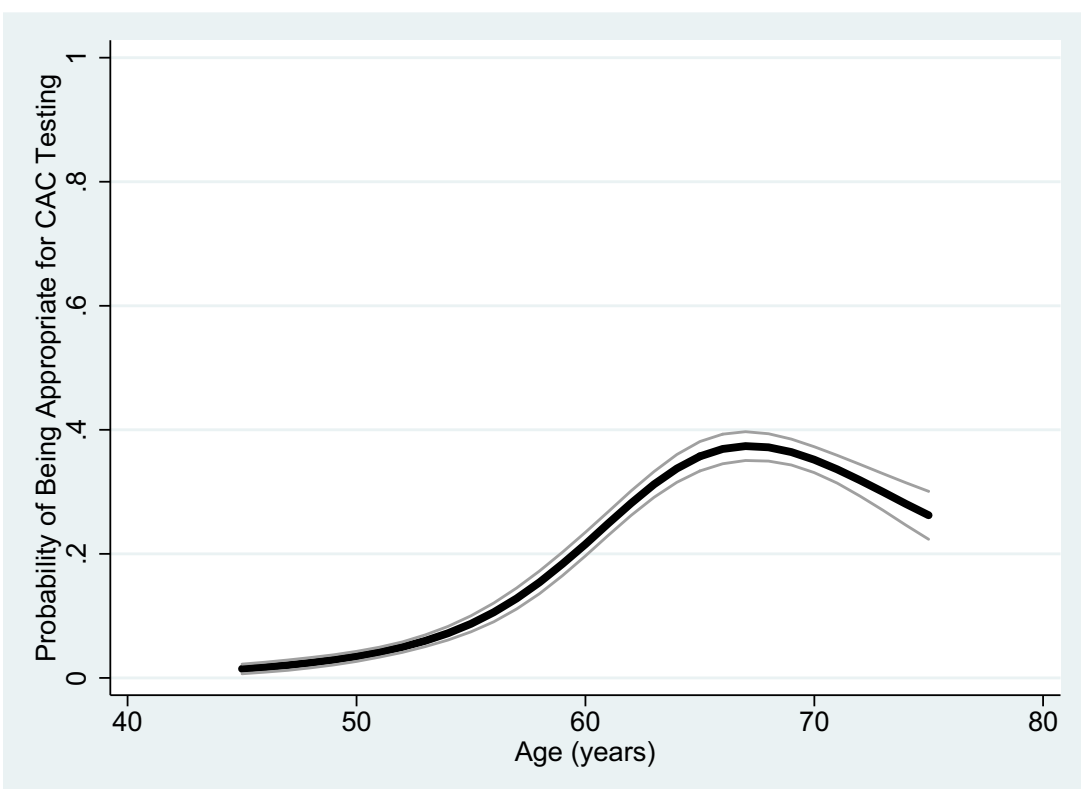

Figure 1 Probability of being appropriate for CAC testing by age of MESA participants.

Corresponding Author: Jeremy B. Sussman, MD, MS; Division of General Medicine University of Michigan, Ann Arbor, MI, USA (e-mail: jeremysu@umich.edu).

Funding Information Dr. Sussman is supported by VA IIR 15-432 and VA CDA 13-021. Dr. Murthy is supported by NIA RO1 AG059729 and NHLBI RO1 HL136685.

\section{Compliance with Ethical Standards:}

This study received exemption from the Michigan Medicine IRB.
Conflict of Interest: Dr. Sussman has no conflicts of interest. Dr. Murthy owns minor stock in General Electric and has research funding from Siemens. Both make scanners used for CAC assessment. Both have received federal grants on topics related to this work.

\section{REFERENCES}

1. Grundy SM, Stone NJ, Bailey AL, et al. AHA/ACC/AACVPR/AAPA/ $\mathrm{ABC} / \mathrm{ACPM} / \mathrm{ADA} / \mathrm{AGS} / \mathrm{APhA} / \mathrm{ASPC} / \mathrm{NLA} / \mathrm{PCNA}$ guideline on the management of blood cholesterol: executive summary: a report of the American College of Cardiology/American Heart Association Task Force on Clinical Practice Guidelines. J Am Coll Cardiol. 2018;S0735-1097(18):3903339038. https://doi.org/10.1016/j.jacc.2018.11.002.

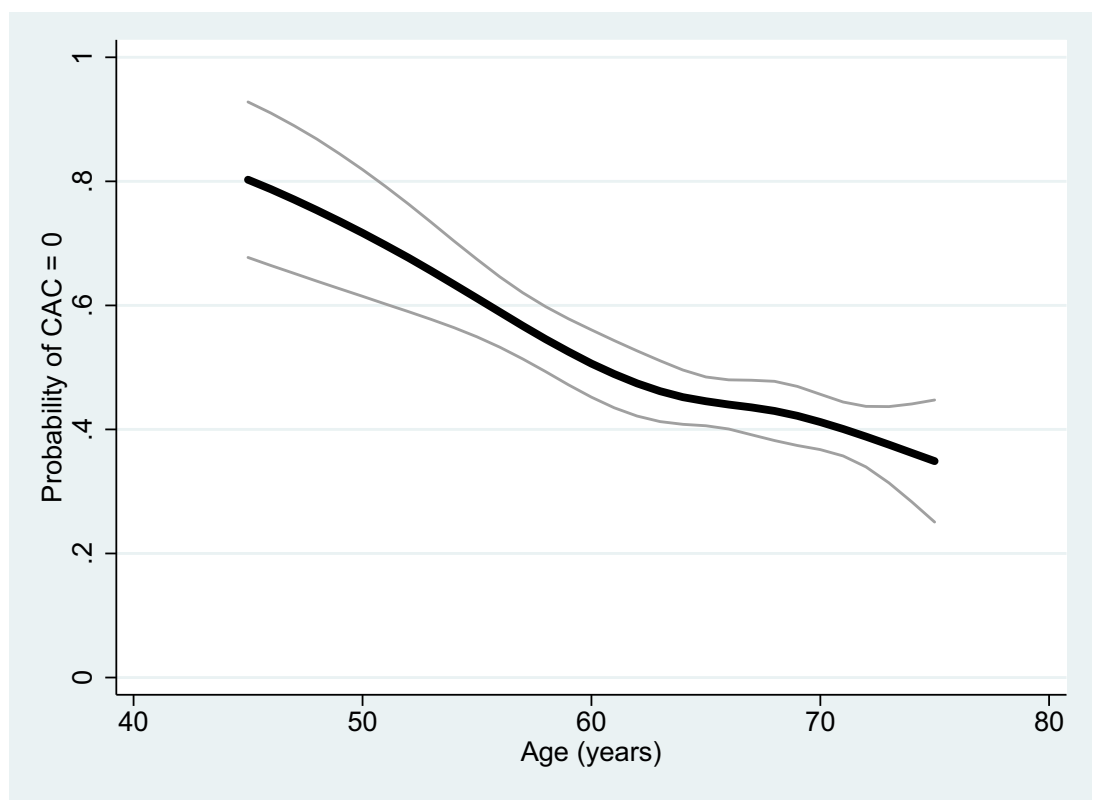

Figure 2 Probability of having a CAC score of 0 by age. 
2. McClelland RL, Chung H, Detrano R, Post W, Kronmal RA. Distribution of coronary artery calcium by race, gender, and age: results from the MultiEthnic Study of Atherosclerosis (MESA). Circulation. 2006;113(1):30-37.

3. McClelland RL, Jorgensen NW, Budoff $\mathbf{M}$, et al. 10-Year Coronary Heart Disease Risk Prediction Using Coronary Artery Calcium and Traditional Risk Factors: Derivation in the MESA (Multi-Ethnic Study of Atherosclerosis) With Validation in the HNR (Heinz Nixdorf Recall) Study and the DHS (Dallas Heart Study). J Am Coll Cardiol. 2015;66(15):16431653.

Publisher's Note Springer Nature remains neutral with regard to jurisdictional claims in published maps and institutional affiliations. 